\title{
Constraining the Collisional Nature of the Dark Matter Through Observations of Gravitational Wakes
}

\section{Citation}

Furlanetto, Steven R., and Abraham Loeb. 2002. "Constraining the Collisional Nature of the Dark Matter through Observations of Gravitational Wakes." The Astrophysical Journal 565 (2): 854-66. https://doi.org/10.1086/324693.

\section{Permanent link}

http://nrs.harvard.edu/urn-3:HUL.InstRepos:41393283

\section{Terms of Use}

This article was downloaded from Harvard University's DASH repository, and is made available under the terms and conditions applicable to Other Posted Material, as set forth at http:// nrs.harvard.edu/urn-3:HUL.InstRepos:dash.current.terms-of-use\#LAA

\section{Share Your Story}

The Harvard community has made this article openly available. Please share how this access benefits you. Submit a story. 


\title{
Constraining the Collisional Nature of the Dark Matter Through Observations of Gravitational Wakes
}

\author{
Steven R. Furlanetto \& Abraham Loeb \\ Harvard-Smithsonian Center for Astrophysics, 60 Garden St., Cambridge, MA 02138; \\ sfurlanetto@cfa.harvard.edu, aloeb@cfa.harvard.edu
}

\begin{abstract}
We propose to use gravitational wakes as a direct observational probe of the collisional nature of the dark matter. We calculate analytically the structure of a wake generated by the motion of a galaxy in the core of an X-ray cluster for dark matter in the highly-collisional and collisionless limits. We show that the difference between these limits can be recovered from detailed X-ray or weak lensing observations. We also discuss the sizes of sub-halos in these limits. Preliminary X-ray data on the motion of NGC 1404 through the Fornax group disfavors fluid-like dark matter but does not exclude scenarios in which the dark matter is weakly collisional.
\end{abstract}

Subject headings: dark matter - X-rays: galaxies: clusters - galaxies: clusters: general - hydrodynamics

\section{Introduction}

Although the dark matter is known to dominate the mass budgets of bound systems such as galaxies or galaxy clusters, its nature remains mysterious. On cosmological scales ( $\gg 1 \mathrm{Mpc}$ ), the standard "cold dark matter" (CDM) model, in which the dark matter is non-baryonic, non-relativistic, and collisionless, has had considerable success matching observations. However, two sets of problems have emerged in applying the same model to smaller scales (Spergel \& Steinhardt 2000; Hogan \& Dalcanton 2000 and references therein). The first is known as the "substructure problem." The CDM model predicts significantly more substructure (in the form of relic dark matter halos) on small scales than is observed. For example, models of the formation of the Local Group of galaxies predict an order of magnitude more dwarf halos than the observed number of dwarf galaxies (Moore et al. 1999). The second problem is known as the "cusp problem": with no interactions to prevent collapse, CDM halos should form nearly singular cores (e.g., Navarro, Frenk, \& White 1997). 
In contrast, observations indicate that galaxies have flat dark matter profiles near their centers (Elores \& Primack 1994; Borriello \& Salucci 2001).

These problems led Spergel \& Steinhardt (2000) to reintroduce self-interacting dark matter (SIDM), originally proposed by Carlson, Machacek, \& Hall (1992; see also de Laix. Scherrer, \& Schaefer 1995). In this scheme, the dark matter has a significant cross-section for self-interaction (and possibly a significant cross-section for interaction with baryons as well; see Kusenko \& Steinhardt 2001). In such a picture, cusp formation is halted by heat conduction (which heats up the dark matter at the halo centers) and the formation of shocks. The substructure problem is avoided because dark matter particle interactions evaporate small halos on relatively short timescales.

Existing limits from laboratory experiments restrict the range of cross-sections available to SIDM that interacts directly with baryons (McGuire \& Steinhardt 2001) but place no restrictions on the cross-section for dark matter-dark matter interactions. To constrain this cross-section, we must turn to astronomical observations. In this paper, we propose such a test, based on observations of the motion of individual galaxies inside galaxy clusters.

As a galaxy moves through a cluster, the gravitational potential of the galaxy induces a wake in the surrounding medium. The back-reaction of this wake on the galaxy is called dynamical friction and is known to be important in the dynamics of cluster galaxies (as well as satellite galaxies and globular clusters on smaller scales; Binney \& Tremaine 1987, Frenk et al. 1996). Wakes are induced in both the dark and gaseous components of the intracluster medium (ICM). The structure of the dark matter wake provides a signature of the collisional nature of the dark matter that is potentially observable through its gravitational lensing effect. The total gravitational potential also imprints a wake in the gas distribution; a precise measurement of the morphology of this wake yields the size of the perturber's dark matter halo. This in turn constrains the collisional nature of the dark matter: the sizes of CDM halos are set by tidal stripping in the cluster potential (Merritt 1988), while ram pressure stripping truncates SIDM halos at considerably smaller radii. The exquisite resolution of the Chandra satellite allows very precise measurements of the extent of surface brightness variations in the ICM gas and could potentially constrain the collisional properties of the dark matter.

In order to approach this problem analytically, we will focus on two extreme scenarios for the nature of the dark matter. The first is the CDM model, in which the dark matter is collisionless. The second model assumes that the dark matter is strongly self-interacting, so that it may be treated as a perfect fluid. We will refer to this scenario as the Fluid Dark Matter (FDM) model. The latter approximation has been used before to isolate the implications of SIDM models (Yoshida et al. 2000; Moore et al. 2000). Our calculations will 
thus bracket the parameter space available for dark matter self-interaction cross-sections. Our experimental test is easiest to interpret in the limit where the perturber mass is much smaller than that of the host cluster, and so we use linear theory in our FDM calculations.

We first outline our formalism for calculating the wake structure induced by a perturber moving through collisionless and collisional media in $\S 2$. We then describe the effects of the ICM dark matter on the perturber halo in $\S 3$. Finally, we present our results and compare them to simulations in $\S 4$, and we discuss their implications in $\S 5$.

\section{Gravitational Wakes Due to Dynamical Friction}

First, we develop the mathematical formalism necessary to calculate the density disturbance in a cluster due to the gravitational effect of a moving galaxy or group of galaxies. In $\S 2.1$ and $\S 2.2$, we calculate the wake in collisionless and collisional media, respectively. In

a collisional medium, an additional wake will be created through deflection of the ambient medium around the surface of the perturber. We discuss this "hydrodynamic wake" in $§ 2.3$. We assume throughout that the perturber (e.g., a galaxy) with mass $M_{g}$ moves through a stationary, uniform background medium (e.g., a cluster core) of density $\rho_{c}$ with a constant velocity $v_{g}=\mathcal{M} \sigma_{c}$ directed along the $z$-axis, where $\mathcal{M}$ is the Mach number of the flow and $\sigma_{c}$ is the velocity dispersion of the background medium (assumed to be equal to the sound speed for simplicity). We include a discussion of when the requirement of a uniform background density may be relaxed in $\S 4$.

\subsection{Collisionless Medium}

First we consider the structure of the wake induced in a collisionless medium, following the method first introduced by Mulder (1983; see also Weinberg 1986). Without collisions, the particle distribution function $f(\mathbf{x}, \mathbf{v})$ must be a function only of the integrals of motion (Binney \& Tremaine 1987), which can be calculated for particles deflected by the perturber using planar orbit theory (Mulder 1983). The precise form of the distribution function is then determined by the boundary conditions: in the frame of the perturber, $f$ must approach a Maxwellian distribution with mean velocity $\mathbf{-} \mathbf{v}_{\mathbf{g}}$ and velocity dispersion $\sigma_{c}$ at infinity. Finally, the density is $\rho=\int f d^{3} \mathbf{v}$. Note that, given the assumption of a uniform background medium, this treatment is exact, and it works equally well for a point mass or an extended body.

To linear order (that is, neglecting the small fraction of background particles that are 
strongly deflected by the perturber), the wake due to a point mass can be treated analytically (Mulder 1983). In the frame of the perturber, the density enhancement $\Delta=(\rho-1) / \rho$ is

$$
\Delta_{\mathrm{CL}, \mathrm{pt}}(\mathbf{x}, t) \approx \frac{G M_{g}}{\sigma_{c}^{2}} \frac{1}{r} \exp \left(-\frac{\mathcal{M}^{2}}{2}\left(1-\cos ^{2} \theta\right)\right)\left[1-\operatorname{erf}\left(\frac{\mathcal{M} \cos \theta}{\sqrt{2}}\right)\right],
$$

where $(r, \theta)$ are polar coordinates centered on the perturber's current location. (Note that Mulder's derivation contains a sign error, though his final expression is correct. We find $\varepsilon \rightarrow-\varepsilon$ in Mulder's equations [AIV.3] and [AIV.4].) This approximation can be derived in

a completely independent manner using the theory of linear response (Colpi \& Pallavicini 1998). Note that the density structure is independent of the propertiers of the perturber and the background medium if we rescale $\tilde{\mathbf{r}}=\mathbf{r} / R_{0}$, where $R_{0}=G M_{g} / \sigma_{c}^{2}$; this is true for an extended perturber as well.

Because of the lack of shocks, wakes in a collisionless medium are smooth regardless of the velocity of the perturber, with the peak density enhancement directly behind the perturber. Increasing the velocity focuses relatively more of the wake behind the perturber, forming a center-filled cone-like structure at high velocities (see Mulder 1983, Fig. 2). This is to be contrasted with the well-defined, edge-filled cones we find below in collisional media.

\subsection{Collisional (Fluid-like) Medium}

We calculate the wake in a highly collisional, or fluid, medium using linear perturbation theory. The passage of the object perturbs the background medium, yielding a density field $\rho=\rho_{c}\left(1+\Delta_{\mathrm{F}}\right)$; we assume that $\Delta_{\mathrm{F}} \ll 1$. The first-order continuity equation is then (see also Ostriker 1999)

$$
\frac{1}{\sigma_{c}} \frac{\partial \Delta_{\mathrm{F}}}{\partial t}+\nabla \cdot \mathbf{v}_{\mathbf{1}}=0
$$

where $\mathbf{v}_{\mathbf{1}}$ is the perturbation to the velocity field, and the Euler equation is

$$
\frac{1}{\sigma_{c}} \frac{\partial \mathbf{v}_{\mathbf{1}}}{\partial t}+\nabla \Delta_{\mathrm{F}}=-\frac{1}{\sigma_{c}^{2}} \nabla \Phi_{\mathrm{ext}}
$$

where $\Phi_{\text {ext }}$ is the gravitational potential of the perturbing body. Combining these equations with Poisson's equation for the potential, eliminating $\mathbf{v}_{\mathbf{1}}$, and Fourier transforming over both space and time yields

$$
\left(k^{2}-\frac{\omega^{2}}{\sigma_{c}^{2}}\right) \hat{\Delta}_{\mathrm{F}}=\frac{4 \pi \mathrm{G}}{\sigma_{c}^{2}} \hat{\rho}_{g} .
$$

Here $\rho_{g}=\rho_{g}\left(R, z-v_{g} t\right)$ is the specified density field of the perturber and a hat denotes a Fourier transformed quantity. This may be simplified by noting that $\hat{\rho}_{g}=(2 \pi)^{1 / 2} \delta(\omega-$ 
$\left.k_{z} v_{g}\right) \hat{\rho}_{0}$, where $\rho_{0}$ is the density distribution of the perturber held stationary at the origin. Taking the inverse Fourier transform and evaluating the Dirac delta function, we find

$$
\Delta_{\mathrm{F}}(\mathbf{x}, t)=\frac{4 \pi \mathrm{G}}{\sigma_{c}^{2}} \int d^{3} \mathbf{k} \frac{\hat{\rho}_{0}}{k^{2}-\mathcal{M}^{2} k_{z}^{2}} e^{i \mathbf{k} \cdot \mathbf{s}}
$$

where $\mathbf{s}=\mathbf{x}-v_{g} t \hat{\mathbf{z}}$. The integral on the right may be inverted with the convolution theorem. However, the inverse Fourier transform of $\hat{f}(\mathbf{k})=1 /\left(k^{2}-\mathcal{M}^{2} k_{z}^{2}\right)$ is not trivial if $\mathcal{M}>1$, because in that case the integrand has poles at $k_{z}= \pm\left(k_{x}^{2}+k_{y}^{2}\right)^{1 / 2} / \beta$, where $\beta^{2}=\mathcal{M}^{2}-1$. We therefore evaluate the integral by transforming to the complex plane and choosing a contour slightly above the real axis. If $s_{z}>0$, the point of interest is ahead of the perturber in real space, and causality dictates that the medium remains unperturbed. We close the contour at $+i \infty$ in this case. Conversely, if $s_{z}<0$, we close the contour at $-i \infty$. Then, using the convolution theorem,

$$
\Delta_{\mathrm{F}}(\mathbf{x}, t)=\frac{G}{\sigma_{c}^{2}} \int d^{3} \mathbf{r}^{\prime} \frac{\xi^{\prime} \rho_{0}\left(\mathbf{r}^{\prime}\right)}{\left[\left(s_{z}-z^{\prime}\right)^{2}-\beta^{2}\left(R^{2}+R^{\prime 2}-2 R R^{\prime} \cos \theta^{\prime}\right)\right]^{1 / 2}},
$$

where

$$
\xi^{\prime}= \begin{cases}2 & \mathcal{M}>1,\left(s_{z}-z^{\prime}\right)+\beta \sqrt{\left(R^{2}+R^{\prime 2}-2 R R^{\prime} \cos \theta^{\prime}\right)}>0 \\ 1 & \mathcal{M}<1, \\ 0 & \text { otherwise. }\end{cases}
$$

In the case of a point mass, $\rho_{0}=M_{g} \delta\left(\mathbf{r}^{\prime}\right)$, this simplifies to the result found by Ostriker (1999):

$$
\Delta_{\mathrm{F}, \mathrm{pt}}(\mathbf{x}, t)=\frac{\xi G M_{g} / \sigma_{c}^{2}}{\sqrt{\left(z-v_{g} t\right)^{2}-\beta^{2} R^{2}}}
$$

where

$$
\xi= \begin{cases}2 & \mathcal{M}>1,\left(z-v_{g} t\right)<-\beta R \\ 1 & \mathcal{M}<1 \\ 0 & \text { otherwise }\end{cases}
$$

As in the collisionless case, the wake structure is independent of the properties of the perturber and the background medium if we rescale $\tilde{\mathbf{s}}=\mathbf{s} / R_{0}$. Note that these formulae are not valid in the case $\mathcal{M}=1$. As shown by Ostriker (1999), the dynamical friction force due to the wake increases rapidly as the velocity approaches the sound speed, so such a perturber quickly slows to subsonic speeds.

In the collisional case, the structure of the wake depends strongly on whether the motion is subsonic or supersonic. In the subsonic case, the wake has front/back symmetry relative to the perturber and varies smoothly. However, in the supersonic case, the wake forms a Mach cone trailing the perturber with opening angle $\cot ^{-1} \beta$. The overdensity at the edges 
of the Mach cone is infinite for a point mass. An extended perturber smooths the singularity, spreading the mass contained in the edge over a region of width $\sim 2 R_{g}$.

For a point mass wake, the column density $\delta \Sigma$ along any sightline intersecting the cone can be computed analytically and is independent of the location in the wake,

$$
\delta \Sigma=\frac{2 \pi \rho_{c} R_{0}}{\sin i\left(\beta^{2}-\cot ^{2} i\right)^{1 / 2}} \quad\left(\cot ^{-1} \beta<i<\pi-\cot ^{-1} \beta\right),
$$

where $i$ is the angle between the direction of motion of the perturber and the line of sight. In the case of an extended perturber, the above formula is very close to the true column density except near the edges of the cone, where the column density drops to zero over a width $\sim 2 R_{g}$.

The singular edge to the cone in the point mass case, and the concentration of mass in this region for an extended mass may raise some concern about the linear perturbation analysis used here. Simulations have shown that, even for a point mass, the resulting dynamical friction force is nevertheless quite accurate (Sánchez-Salcedo \& Brandenburg 1999), so we expect the linear treatment to work adequately. We do find that $\Delta_{F}<1$ throughout most of the volume of interest (far from the perturber), so linear theory should be accurate in these regions. We include a more in-depth discussion of existing simulations in $\S 4.3$.

\subsection{Hydrodynamic Wake}

For a collisional medium, we must add the hydrodynamic disturbance introduced by the passage of the perturber to the gravitational wake. If the bound halo is "stiff," it deflects ICM particles and sends pressure perturbations into the ambient medium. The hard surface approximation is expected to be adequate for the ICM gas, because the Larmor radius for typical cluster magnetic fields is very small ( $\ll 1 \mathrm{pc})$, although particles may still penetrate the perturber if the field lines intersect the body. The width of the edge of the dark matter halo is expected to be of order the dark matter particle mean free path.

The density disturbance due to the surface effect may be calculated analytically far from the perturber if the perturbing body is streamlined (Landau \& Lifshitz 1959, §115). We switch to the rest frame of the perturber and use coordinates $\mathbf{s}$ with the origin at the center of the body. In this frame, the gas velocity is $\mathbf{v}_{\mathbf{0}}=-v_{0} \hat{\mathbf{s}}_{\mathbf{z}}+\mathbf{v}_{\mathbf{1}}$ and the density is $\rho=\rho_{c}\left(1+\Delta_{\mathrm{HD}}\right)$, where $\Delta_{\mathrm{HD}}$ is the density perturbation due to this hydrodynamic effect. Far from the perturbing galaxy, the gas is only slightly disturbed and linear theory applies. If we assume that $\mathbf{v}_{\mathbf{1}}$ is related to a velocity potential $\phi$ via $\mathbf{v}_{\mathbf{1}}=\nabla \phi$, the Euler equation 
may be written as

$$
\Delta_{\mathrm{HD}}=-\frac{v_{0}}{\sigma_{c}^{2}} \frac{\partial \phi}{\partial s_{z}}
$$

where we have assumed that $\phi$ and $\Delta_{\mathrm{HD}}$ vanish at infinity. Using the continuity equation to eliminate the density enhancement, we find

$$
\frac{\partial^{2} \phi}{\partial s_{x}^{2}}+\frac{\partial^{2} \phi}{\partial s_{y}^{2}}-\beta^{2} \frac{\partial^{2} \phi}{\partial s_{z}^{2}}=0
$$

The solution is (Landau \& Lifshitz 1959)

$$
\phi=-\frac{v_{0}}{2 \pi} \int_{C}^{\ell} \frac{S^{\prime}(u) d u}{\sqrt{\left(s_{z}-u\right)^{2}-\beta^{2} R^{2}}},
$$

where $C=\max \left(s_{z}+\beta R,-\ell\right), 2 \ell$ is the length of the perturbing body in the direction of motion, $S(z)$ is the cross-section of the perturbing body, and a prime represents differentiation with respect to $u$. The length $\ell$ of the body is not well-determined in our analysis; the linear theory is strictly only valid for bodies with very small opening angles. We let $\ell=x R_{g}$, where $R_{g}$ is the transverse radius of the object (see $\S 3$ ). Note, however, that the assumption of a streamlined perturber is not likely to be valid in realistic circumstances.

In this limit, the hydrodynamic wake forms a cone with a structure similar to the gravitational wake. Both are "edge-filled" cones with opening angle $\cot ^{-1} \beta$. In the approximation used above, weak discontinuities appear at the Mach cones originating from the leading and trailing edges of the body; in reality, far from the perturber, these are weak shocks (Landau \& Lifshitz 1959; §114). The ICM gas is compressed at the leading shock and then becomes rarefied as it deflects around the body. The trailing shock discontinuously increases the gas density. As equation (13) shows, the density distribution of the wake depends on the cross-section of the body, which is not well-determined by our treatment (it depends on the detailed initial mass distribution, gas replenishment in the galaxy, and the nature of the ram-pressure stripping). Unfortunately, the dependence on the cross-section is substantial, particularly between the two discontinuities. In addition, real halos are not streamlined (Markevitch et al. 2000; Vikhlinin, Markevitch, \& Murray 2001), but rather have blunt surfaces. In this case the leading shock is "detached" from the front of the halo. The bow shock is normal to the direction of motion on the collision axis and asymptotically approaches the Mach cone at large distances (Schreier 1982). Within this region, the hydrodynamic motions are quite complex, but some general conclusions can be drawn.

Behind the trailing shock, the density enhancement due to the perturbing body falls off rapidly. This is best illustrated by evaluating equation (13) for $R \ll-s_{z}$. We find

$$
\Delta_{\mathrm{HD}} \approx \eta x \mathcal{M}^{2}\left(\frac{R_{g}}{\left|s_{z}\right|}\right)^{3},
$$


where $\eta \sim 1$ depends on the cross-section of the perturber. We find that $\Delta_{\mathrm{HD}} \ll 1$ throughout most of the cone interior. However, the compressions and rarefactions are strongest in the region bounded by the two shocks discussed above. We find that in this region

$$
\Delta_{\mathrm{HD}} \approx \frac{\mathcal{M}^{2}}{x^{2}} \sqrt{\frac{-s_{z}-\beta R+x R_{g}}{2 \beta R}} p\left(\frac{-s_{z}-\beta R+x R_{g}}{2 x R_{g}}\right) \quad-x R_{g}<s_{z}+\beta R<x R_{g} .
$$

Here $p(y)$ is a function that depends on the precise form of the perturber's cross-section; in general, it changes sign as $R$ varies. Because $s_{z}+\beta R$ is bounded, in this region $\delta \Sigma_{\mathrm{HD}} \propto$ $\Delta_{\mathrm{HD}} \propto\left|s_{z}\right|^{-1 / 2}$. We find for some generic cross-sections that $\left|\Delta_{\mathrm{HD}}\right|>1$ within the region of interest, indicating that linear theory is not valid. Therefore we cannot meaningfully compare the calculated amplitudes of the variations in this region to the gravitational wake without detailed simulations of the interaction process. However, note that the integrated mass perturbation along any line of sight passing near the collision axis $\delta \Sigma_{\mathrm{HD}}$ vanishes. As the body moves, it produces finite outgoing cylindrical sound waves. The total displaced mass through any line of sight must therefore vanish (Landau \& Lifshitz 1959, §95).

There is yet another effect that can create a wake in a collisional medium. Stevens, Acreman, \& Ponman (1999) simulated the effects of ram pressure stripping on the interstellar medium of galaxies moving through the ICM. Most of the gas is stripped into a plume trailing the galaxy, but they also found "bow shock" wakes with similar structure to those produced by point masses. The simulation box size was comparable to the size of the dark matter distribution of the galaxy, and so it is not possible to infer directly the effects of the stripped gas on large scales $\left(\gg R_{\text {gas }}\right.$ ) which are of interest here. The finite mass of gas in the galaxy (particularly after it has traveled some distance through the cluster) indicates that the stripped gas should not have an appreciable effect on the large-scale wake structure (see $\S 3.2$ below).

In summary, we expect that hydrodynamic interactions around the perturber will induce a wake of similar structure to the gravitational wake in a collisional medium. Although the overdensity in the interior of the hydrodynamic wake falls off quickly with distance from the perturber, the mass between the shocks produced by the leading and trailing edges of the pertuber decreases much more slowly and, in fact, linear theory breaks down for a large region around the perturber. Therefore we cannot put meaningful constraints on the observability of the hydrodynamic wake.

\section{Halo Truncation in Galaxy Clusters}




\subsection{Dark Matter Stripping Processes}

Next we describe how to apply the above formalism to galaxies and groups moving through X-ray clusters. We assume that the perturber moves through a constant density cluster core, surrounded by a softened isothermal envelope

$$
n(r)= \begin{cases}n_{c} & r<r_{c} \\ 2 n_{c} /\left[1+\left(r / r_{c}\right)^{2}\right] & r \geq r_{c}\end{cases}
$$

where $n_{c}$ is the total number density of ions and electrons in the core and $r_{c}$ is the core radius. For simplicity, we assume the dark matter distribution in the perturber to be a singular isothermal sphere with a specified velocity dispersion $\sigma_{g}$, so that $\rho_{g}=\sigma_{g}^{2} /\left(2 \pi G r^{2}\right)$. The mass contained within a radius $R_{g}$ is then $M_{g}=2 \sigma_{g}^{2} R_{g} / G$.

The radius of the halo is determined by the nature of the dark matter. If the dark matter is collisionless, only gravitational variations within the cluster can affect the halo. Thus tidal stripping determines the final size, and the truncation radius may be found by solving $\bar{\rho}_{g}\left(<R_{g}\right)=\rho_{c}$, where $\bar{\rho}_{g}\left(<R_{g}\right)$ is the mean density inside $R_{g}$ (Babul \& Taylor 2000), as also implied by numerical simulations (Merritt 1988). If the dark matter is strongly selfinteracting, the halo is subject to ram pressure stripping as well. The truncation radius is then determined approximately by balancing the ram pressure and the external pressure, $\rho_{c} v_{g}^{2}=\rho_{g}\left(R_{g}\right) \sigma_{g}^{2}$. This simple prescription ignores two competing effects. First, the supersonic motion of the perturber creates a bow shock leading it (see §2.3), which shields the halo from the ICM ram pressure. However, simulations show that for mildly supersonic motion the bow shock provides relatively little protection (Stevens et al. 1999; Quilis et al. 2000). Instead, recent high-resolution simulations of galaxy motion through the ICM show that turbulent and viscous stripping are as effective as ram pressure in truncating gas disks (Quilis et al. 2000). More realistic prescriptions will therefore only increase the contrast between the FDM and CDM model predictions. Solving for $R_{g}$ in the two cases yields

$$
R_{g}=164\left(\frac{5 \times 10^{-3} \mathrm{~cm}^{-3}}{n_{c}} \frac{f_{g}}{0.2} \frac{0.6}{\mu}\right)^{1 / 2}\left(\frac{\sigma_{g}}{300 \mathrm{~km} \mathrm{~s}^{-1}}\right) \alpha_{\mathrm{DM}} \mathrm{kpc}
$$

where

$$
\alpha= \begin{cases}(\sqrt{3} \mathcal{M})^{-1}\left(\sigma_{g} / \sigma_{c}\right), & \text { collisional medium, } \\ 1, & \text { collisionless medium, }\end{cases}
$$

$f_{g}$ is the gas-to-dark matter mass ratio in the cluster, $\mu$ is the mean atomic weight per particle, and the subscript "DM" refers to the dark matter medium. The length scale $R_{0}$ is

$$
R_{0}=30\left(\frac{5 \times 10^{-3} \mathrm{~cm}^{-3}}{n_{c}} \frac{f_{g}}{0.2} \frac{0.6}{\mu}\right)^{1 / 2}\left(\frac{\sigma_{g} / \sigma_{c}}{0.3}\right)^{2}\left(\frac{\sigma_{g}}{300 \mathrm{~km} \mathrm{~s}^{-1}}\right) \alpha_{\mathrm{DM}} \mathrm{kpc} .
$$


Note that the scaled size of the perturber is

$$
\tilde{R}_{g} \equiv \frac{R_{g}}{R_{0}}=\frac{1}{2}\left(\frac{\sigma_{c}}{\sigma_{g}}\right)^{2} .
$$

Studies of galaxies in nearby clusters, particularly Virgo and Coma, have shown convincing evidence for ram pressure stripping of the interstellar atomic gas (Warmels 1988; Cayatte et al. 1994). Magri et al. (1988) showed that the HI flux from spiral galaxies increases with distance from the cluster center and decreases with the X-ray luminosity of the host cluster, as one would expect for ram pressure stripping. Most recently, Bravo-Alfaro et al. (2000) examined 19 bright spiral galaxies in the Coma cluster and found a clear correlation between depth in the cluster and HI deficiency relative to isolated spirals. Rubin, Waterman, \& Kenney (1999) reach a similar conclusion for the ionized gas disks of spirals in the Virgo cluster.

It is much less clear whether dark matter may be stripped in these environments. Despite early evidence showing that cluster galaxies tend to have falling rotation curves (Whitmore, Forbes, \& Rubin 1988), more recent studies have not found any systematic difference in the shape of the curves with location in the cluster (Dale et al. 2001, and references therein). However, these observations do not rule out the FDM model, because the observed rotation curves are based on $\mathrm{H} \alpha$ emission. This gas is subject to ram pressure and is therefore at least as compact as the dark matter. A better test is to examine the stellar rotation curves, which at these distances can only be probed with bright tracers such as planetary nebulae (Ciardullo, Jacoby, \& Dejonghe 1993; Arnaboldi et al. 1996; Arnaboldi et al. 1998). To date, such studies have been able to examine the inner $\sim 20 \mathrm{kpc}$ of relatively massive ellipticals, about twice the extent of the emission line rotation curves. The observations show no evidence for deviations from the normal flat rotation curves; however, their limited extent would not exclude a scenario that is only somewhat less efficient than our FDM model.

Another limit comes from the fact that as the halo loses its dark matter, luminous matter in the galaxy becomes unbound. As a simple model, we consider a spherical galaxy with both the dark matter and stars distributed as singular isothermal spheres. In this case the distribution of stellar velocities is Maxwellian with standard deviation $\sigma_{g}$ (Binney \& Tremaine 1987). We suppose that the initial radius of stellar matter is $R_{i}$. After stripping, the dark matter radius is reduced to $R_{g}$; if $R_{g}<R_{i}$, we assume that stars outside of $R_{g}$ can escape from the galaxy if their total energy is positive. The fraction of stars that escape the galaxy is then

$$
f_{\mathrm{esc}}=\frac{R_{g}}{R_{i}} \int_{1}^{R_{i} / R_{g}} \operatorname{erfc}\left(\sqrt{\frac{2}{r}}\right) d r .
$$


Using the observed relation between the velocity dispersion of a halo and its effective radius $R_{e}$ (Binney \& Merrifield 1998, p. 206 ), we find that in the FDM model

$$
\frac{R_{i}}{R_{g}} \approx 1.1 \mathcal{M}\left(\frac{5 \times 10^{-3} \mathrm{~cm}^{-3}}{n_{c}} \frac{f_{g}}{0.2} \frac{0.6}{\mu}\right)^{-1 / 2}\left(\frac{\sigma_{g}}{200 \mathrm{~km} \mathrm{~s}^{-1}}\right)^{-0.2}\left(\frac{\sigma_{c}}{1000 \mathrm{~km} \mathrm{~s}^{-1}}\right),
$$

where we have set $R_{e}=0.36 R_{i}$ for a singular isothermal sphere. The well-known "fundamental plane" relates the luminosity (assumed proportional to the number of stars), the effective radius, and the velocity dispersion of ellipticals. The scatter in the relation is only $\sim 0.07$ dex (Mobasher et al. 1999). Because the mass loss mechanism described above moves galaxies away from the fundamental plane, the scatter implies that $R_{i} / R_{g} \lesssim 1.2$ for galaxies in clusters, or that $\mathcal{M} \lesssim 1.1$ in typical rich clusters. However, note that we have neglected the contribution of the (non-stripped) stars to the mass of the galaxy, which may be substantial in the inner regions. This limit is therefore currently no stronger than that provided by rotation curves of galaxies.

On the other hand, observations of dwarf galaxies in the Local Group indicate that dark matter stripping may be much more effective than the CDM model predicts. At least three dwarf spheroidals (Carina, Draco, and Ursa Minor) are observed to have stellar tails outside of their optical cutoff radii (Irwin \& Hatzidimitriou 1995). Significant amounts of dark matter outside of the optical radii would preclude escape of these stars, indicating that $R_{g} \sim 300 \mathrm{pc}$ for these systems (Moore 1996; Burkert 1997). Our model predicts $R_{g, \mathrm{CDM}} \sim 5 \mathrm{kpc}$ and $R_{g, \text { FDM }} \sim 200$ pc for a typical dwarf galaxy with $\sigma_{g} \sim 10 \mathrm{~km} \mathrm{~s}^{-1}$, favoring a model that is "nearly" FDM. One caveat is that, if the orbits of the dwarf galaxies are highly eccentric, tidal stripping may be much more effective than is inferred from their present locations.

Heat conduction from the hot ICM to the bound halo dark matter will also tend to evaporate the halos. However, the thermal conductivity of a medium is $\kappa \propto \lambda$, where $\lambda$ is the mean free path of the particles. In the extreme FDM limit, $\lambda=0$ and no heat conduction occurs; Gnedin \& Ostriker (2000) find that heat conduction may be neglected over a Hubble time if $\sigma_{\mathrm{DM}} / m_{\mathrm{DM}} \gtrsim 10^{4} \mathrm{~cm}^{2} \mathrm{~g}^{-1}$, where $\sigma_{\mathrm{DM}}$ and $m_{\mathrm{DM}}$ are the self-interaction cross-section and mass of the dark matter particles, respectively. If $\lambda \neq 0$, heat conduction and ram pressure will work together to truncate halos, making that process more efficient than we would otherwise expect. In the opposite regime of very weakly interacting dark matter particles, scattering interactions between halo and cluster particles can also evaporate the halo. However, in the CDM limit, this process can be neglected. Gnedin \& Ostriker (2000) find that the evaporation time is greater than a Hubble time for $\sigma_{\mathrm{DM}} / m_{\mathrm{DM}} \lesssim 0.3 \mathrm{~cm}^{2} \mathrm{~g}^{-1}$. Note that evaporation operates independently of the motion of the galaxy through the ICM, and it will therefore truncate all subhalos, even those of central cD galaxies. 


\subsection{Indirect Effects of Dynamical Friction in Clusters}

A common difficulty in all treatments of dynamical friction in a uniform infinite medium is that the mass contained in the wake diverges with distance from the perturber. This divergence is normally avoided by assuming that the perturber is embedded in a medium of finite extent (Binney \& Tremaine 1987). The divergence implies that there must exist

a distance $\tilde{r}_{\text {wake }}$ inside of which the wake contains mass $M_{g}$. Beyond $\tilde{r}_{\text {wake }}$, we expect the second-order effect of the dark matter wake structure on the observed medium to dominate the effect induced by the perturber itself. Using the analytic approximations for the wake due to a point mass, we find

$$
\tilde{r}_{\text {wake }} \approx 15 \alpha_{\mathrm{DM}}^{-1}\left(\frac{\sigma_{g} / \sigma_{c}}{0.3}\right)^{-3} g(\mathcal{M})^{-1 / 2} .
$$

where

$$
g(\mathcal{M})= \begin{cases}2 /\left(\mathcal{M}^{2}-1\right), & \text { FDM } \\ \int_{-1}^{1} \exp \left[-\frac{1}{2} \mathcal{M}^{2}\left(1-\mu^{2}\right)\right][1-\operatorname{erf}(\mathcal{M} \mu / \sqrt{2})] d \mu, & \text { CDM }\end{cases}
$$

In the cases we study, we find that $\tilde{r}_{\text {wake }}$ is comparable to or somewhat greater than the box size of our calculations (and thus larger than cluster cores; see §4). Therefore, we neglect the effect of the dark matter wake on itself and on the gas wake. We have confirmed numerically that the dark matter wake has only a small effect on its own structure within $\tilde{r}_{\text {wake }}$. We can also examine the extent of the stripped gas wake. If we let $M_{\text {gas }}=k M_{g}$ and assume that the stripped gas forms a wake similar to the one we have calculated, the stripped gas will be contained within a distance $\tilde{r}_{\text {gas }} \sim k^{1 / 2} \tilde{r}_{\text {wake }} \ll \tilde{r}_{\text {wake. }}$. Thus the stripped gas should be confined to the central region around the perturber.

Note that the dynamical friction force $F_{\mathrm{DF}} \propto M_{g}^{2}$ in both the CDM and FDM models, with the coefficient $I_{\mathrm{DF}}$ varying by a factor of a few between the two (Ostriker 1999). Because FDM halos are less massive than CDM halos by a factor of $\alpha_{\mathrm{FDM}}$, we expect that FDM halos will lose energy to dynamical friction at a slower rate than CDM halos and will therefore sink to the cluster core at a slower rate. However, FDM halos experience a ram pressure force $F_{\text {ram }}$ as well. In our model, $F_{\mathrm{DF}} / F_{\text {ram }} \approx 4 I_{\mathrm{DF}} /\left(\mathcal{M}^{4} \tilde{R}_{g}^{2}\right) \ll 1$, so the FDM sink time is determined primarily by ram pressure. The different causes of sinking in the two regimes can lead to observable effects in the galaxy distribution (Moore et al. 2000). In particular, if ram pressure is effective, the time for a galaxy originally situated a radius $r_{b}$ from the cluster center to sink to the center is $t_{\mathrm{ram}} \sim 4 r_{b} / \sigma_{c}$. This is generally much smaller than the sink time for CDM halos; we find

$$
\frac{t_{\mathrm{DF}, \mathrm{CDM}}}{t_{\mathrm{ram}, \mathrm{FDM}}} \sim \frac{4.4 \mathcal{M}^{3}}{I_{\mathrm{DF}}}\left(\frac{\sigma_{c} / \sigma_{g}}{5}\right)^{3} .
$$


Like Moore et al. (2000), we find that the sink time in the FDM regime is independent of the properties of the galaxy. In contrast, the sink time in the CDM regime has a fairly steep dependence on $\sigma_{c} / \sigma_{g}$, implying that in a given cluster we should observe the massive galaxies to be more dynamically relaxed than smaller galaxies. Such mass (or luminosity) segregation has been observed, although its origins are controversial because morphology segregation in clusters can mask intrinsic mass segregation (Kashikawa et al. 1998 and references therein). Kashikawa et al. (1998) have found that brighter galaxies are more centrally concentrated than faint galaxies in Coma, though the degree of concentration depends on morphology. Furthermore, Drinkwater, Gregg, \& Colless (2001) found that the velocity dispersion of the giant galaxies in the Fornax group is a factor of $\sqrt{2}$ smaller than the velocity dispersion of the dwarf galaxies, indicating that the former are in a more relaxed state. This may be due to the more rapid effects of dynamical friction on larger galaxies. A similar effect has been observed for galaxies in rich clusters (Adami, Biviano, \& Mazure 1998).

A further observable effect that we have not included in our calculations is the possibility of a separation between the dark and gaseous components of the perturber. In the FDM case, the two components are subject to the same decelarating forces (ram pressure and dynamical friction), but a CDM halo feels only dynamical friction. We therefore expect CDM halos to lead gaseous halos by an amount that depends on the time history of the interaction. Approximate equilibrium can be reached if the gravitational attraction between the dark matter halo and the gas halo balances the ram pressure experienced by the gas halo (note, however, that such a separation may be convectively unstable; Ricker \& Sarazin 2001). We can estimate this distance if we ignore other forces (including gradients in the cluster potential); we then find that the equilibrium separation is

$$
D_{\text {eq }}=86\left(\frac{\gamma}{\mathcal{M}} \frac{5 \times 10^{-3} \mathrm{~cm}^{-3}}{n_{c}} \frac{0.6}{\mu}\right)^{1 / 2}\left(\frac{f_{g}}{0.2}\right)\left(\frac{\sigma_{g} / \sigma_{c}}{0.3}\right)^{1 / 2}\left(\frac{\sigma_{g}}{300 \mathrm{~km} \mathrm{~s}^{-1}}\right) \mathrm{kpc}
$$

where $\gamma \sim 1$ depends on the structure of the gas and dark matter halos. Such a separation has been observed between the galaxies in the NGC 4839 group and its intragroup gas as the group falls into the Coma cluster, and it can be attributed to the much smaller ram pressure experienced by the compact galaxies (Neumann et al. 2001). The cluster parameters of Neumann et al. and the observed values of $\sigma_{g} / \sigma_{c}=0.3$ and $\mathcal{M} \approx 1.7$ (Colless \& Dunn 1996) suggest an equilibrium separation of $D_{\text {eq }} \sim 1 \mathrm{Mpc}$, much larger than the observed separation of $\sim 200 h^{-1} \mathrm{kpc}$, where the Hubble constant $H_{0}=100 h \mathrm{~km} \mathrm{~s}^{-1} \mathrm{Mpc}^{-1}$. This is consistent with other suggestions that the merger is recent, with the NGC 4839 group on its first infall into Coma. Nevertheless, combining the X-ray map with a weak lensing map in this or a similar system could constrain the collisional nature of the dark matter simply by locating the mass peak. 


\section{Results}

Two techniques may be employed to observe the effects of the truncated dark matter halos. First, gravitational lensing can constrain the projected surface mass density of the perturber/cluster system (§4.1). Alternatively, the wake in the ICM gas can be observed through its X-ray bremsstrahlung emission ( $\$ 4.2)$. The former method has the advantage of directly testing the dark matter profile, but the X-ray signal is stronger and easier to observe.

In presenting our results, we must ensure that our formalism (in particular the assumption of a constant density background medium) remains valid. If the background medium has $\rho_{0}=\rho_{0}(R)$, where $R$ is radial distance in the cluster, we have

$$
\nabla^{2} \Delta_{\mathrm{F}}-\frac{1}{\sigma_{c}^{2}} \frac{\partial^{2} \Delta_{\mathrm{F}}}{\partial t^{2}}=-\frac{1}{\rho_{0}} \frac{\partial \rho_{0}}{\partial R}\left[\frac{\partial \Delta_{\mathrm{F}}}{\partial R}-\frac{1}{\sigma_{c}^{2}} \frac{\partial \Phi_{\mathrm{ext}}}{\partial R}\right]-\frac{1}{\sigma_{c}^{2}} \nabla^{2} \Phi_{\mathrm{ext}}
$$

in place of the wave equation with a single source term that led to equation (4). We expect that $\left(\partial \Delta_{\mathrm{F}} / \partial R\right) \sim \sigma_{c}^{-2}\left(\partial \Phi_{\text {ext }} / \partial R\right) \sim 1 / R_{0}$ while $\rho_{0}^{-1}\left(\partial \rho_{0} / \partial R\right) \sim 1 / r_{c}$. Therefore, in order to neglect the bracketed term on the right-hand side, we require $r_{c} / R_{0} \equiv \tilde{r}_{c} \gg 1$. A proper treatment in regions with rapidly varying density (as well as an examination of the effects of shocks and other nonlinearities) requires numerical simulations. For these reasons, we discuss our results in light of existing simulations in $\S 4.3$.

\subsection{Projected Surface Mass Density}

The top two panels of Figure 1 show the surface density of a $\sigma_{g}=500 \mathrm{~km} \mathrm{~s}^{-1}$ group merging with the core of a $\sigma_{c}=1000 \mathrm{~km} \mathrm{~s}^{-1}$ cluster with $n_{c}=5.4 \times 10^{-3} \mathrm{~cm}^{-3}$ and $r_{c}=$ $0.4 \mathrm{Mpc}$. The group has $\mathcal{M}=2$. The top left panel shows the surface density for the FDM model, and the top right panel shows the corresponding result for the CDM model. The horizontal dashed lines in each panel mark where the Mach cone with its apex at the center of the perturber intersects the edge of the cluster core; the apparent suppression of the signal below this line occurs only because we do not allow the wake to propagate out of the cluster core.

The most straightforward way to detect the FDM Mach cone is to look for the abrupt drop in projected mass at its edge. The strength of this signal can be estimated by comparing the (constant) surface density of the cone, given by equation (8), to the surface density of the cluster in which it is embedded, $\Sigma$. In the limit in which the impact parameter of the 


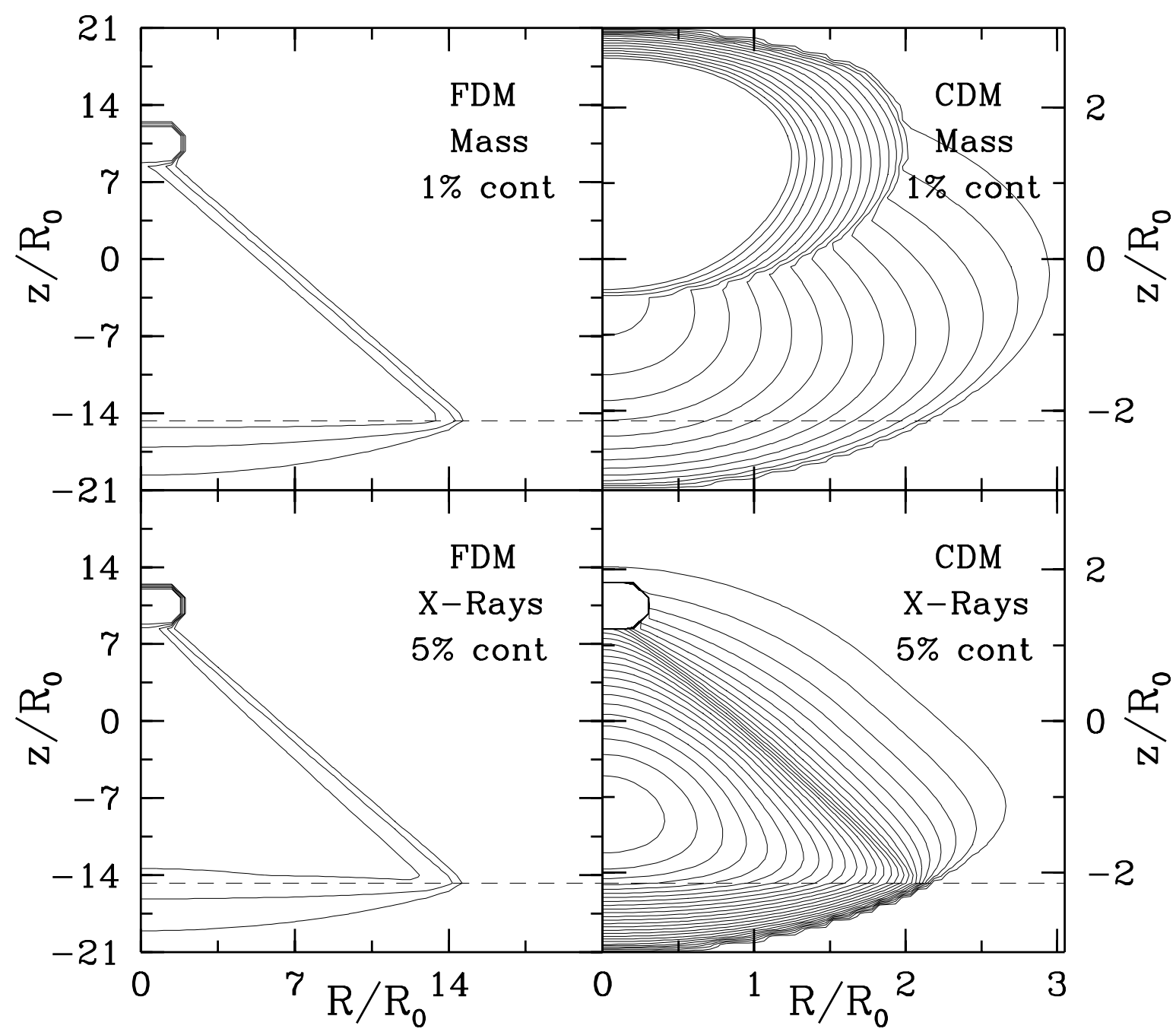

Fig. 1.- Results for a $\sigma_{g}=500 \mathrm{~km} \mathrm{~s}^{-1}$ perturber moving with $\mathcal{M}=2$ through a background cluster with $\sigma_{c}=1000 \mathrm{~km} \mathrm{~s}^{-1}, n_{c}=5.4 \times 10^{-3} \mathrm{~cm}^{-3}$ and $r_{c}=0.4 \mathrm{Mpc}$. Top left: Projected mass profile, FDM model. Top right: Projected mass profile, CDM model. Bottom left: Surface brightness, FDM model. Bottom right: Surface brightness, CDM model. Contours are set at $1 \%$ of the peak surface density of the background cluster in the top panels and at $5 \%$ of the peak surface brightness of the background cluster in the bottom panels. The merger occurs in the plane of the sky with the perturber moving along the $z$-axis. Axes (in cylindrical coordinates) are in units of $R_{0}$ appropriate to each panel (see Eq. [19]). Horizontal dashed lines mark the intersection of the Mach cone with the cluster core. 
line of sight is much less than $r_{c}$, the excess is

$$
\left.\frac{\delta \Sigma}{\Sigma}\right|_{\mathrm{FDM}} \sim\left(\frac{\pi}{\pi / 2+1}\right) \frac{\tilde{r}_{c}^{-1}}{\sin i\left(\beta^{2}-\cot ^{2} i\right)^{1 / 2}} \quad\left(\cot ^{-1} \beta<i<\pi-\cot ^{-1} \beta\right) .
$$

Here

$$
\tilde{r}_{c}=13.3\left(\frac{r_{c}}{400 \mathrm{kpc}}\right)\left(\frac{5 \times 10^{-3} \mathrm{~cm}^{-3}}{n_{c}} \frac{f_{g}}{0.2} \frac{0.6}{\mu}\right)^{-1 / 2}\left(\frac{\sigma_{g} / \sigma_{c}}{0.3}\right)^{-2}\left(\frac{\sigma_{g}}{300 \mathrm{~km} \mathrm{~s}^{-1}}\right)^{-1} \alpha_{\mathrm{DM}}^{-1} .
$$

The amplitude depends strongly on the ratio of the velocity dispersions, so the best candidates are galaxies moving through groups or groups of galaxies moving through clusters. The small surface density of groups makes lensing observations difficult, so we focus on the latter possibility in this section.

As discussed in $§ 2.3$, collisional media also give rise to wakes from hydrodynamic and stripping processes. The effects of the hydrodynamic wake well behind the perturber should be small, because $\delta \Sigma_{\mathrm{HD}}$ vanishes to linear order. However, the stripped dark matter could enhance the signal immediately behind the galaxy and the leading bow shock may be quite strong as well; we therefore regard our calculation as a lower limit to the true enhancement in the FDM case.

Another observable difference between the two pictures is the amplitude of the mass perturbation induced by gravity. Tidal stripping in the CDM model leaves halos much more extended than FDM halos. They are therefore more massive and have correspondingly more gravitational influence on the ambient medium; equation (28) shows that $\delta \Sigma / \Sigma \propto \alpha_{\mathrm{DM}}$. In fact, the enhancement in Figure 1 is somewhat smaller than this because the CDM wake does not have the same structure as the FDM wake.

A third observable effect is the size of the dark matter halo itself, which is dramatically different in the two regimes because of the different stripping mechanisms. By correlating the mass maps (from lensing) with X-ray maps, we can compare the relative sizes of the gas halo (which we know to be stripped by ram pressure) and the dark matter halo. One method is that of Natarajan \& Kneib (1997), who analyzed the variation in the shear of background galaxies in concentric annuli around cluster galaxies in order to constrain the sizes of their dark matter halos. So far, the method has yielded only an upper limit on the sizes of the halos that is comparable to their tidal radius (Natarajan et al. 1998). Such a comparison should be more straightforward in the case of a group of galaxies merging with a larger cluster. Note that this effect does not necessarily rely on the extreme FDM model, because a combination of ram pressure, heat conduction, and evaporation due to scattering will occur across the entire possible range of parameters for SIDM. 
The recent Chandra images of sharply defined gas halos in merging clusters offer excellent opportunities for such comparisons. In particular, Markevitch et al. (2000) found two sharp X-ray surface brightness features in the merging cluster Abell 2142. They argue that these edges are formed by ram-pressure stripping during an ongoing merger in which the two components have passed each other once. Optical data indicate that the merger velocity is $\mathcal{M} \sim 1.5$ and that $\sigma_{c} \sim 1200 \mathrm{~km} \mathrm{~s}^{-1}$ (Oegerle, Hill, \& Fitchett 1995). The smaller component has $R_{\text {gas }} \sim 50 h^{-1} \mathrm{kpc}$. Given the observed parameters of the cluster gas Henry \& Briel 1996), equation (17) suggests that the velocity dispersion of the smaller component is $\sigma_{g} \sim 700 \mathrm{~km} \mathrm{~s}^{-1}$. Our FDM model therefore predicts a mass enhancement $\delta \Sigma / \Sigma \sim 11 \%$ behind the smaller subcluster. In the CDM model, we predict a more gradual density increase with a peak amplitude $\delta \Sigma / \Sigma \sim 45 \%$, although the system is at the limit of the validity of linear theory. Brightness edges similar to these have been observed in several other clusters (Vikhlinin, Markevitch, \& Murray 2001; Sun et al. 2001), indicating that such events are not uncommon.

An alternate approach to tracing the mass distribution around the subhalo is to map the distribution of nearby dwarf galaxies (Markevitch \& Vikhlinin 2001, private communication). Dwarfs behave as collisionless test particles and so form a wake similar in structure to that shown in the top right panel of Figure 1. The amplitude of the variation in surface density of dwarfs near galaxies and groups can then be used to constrain the mass of the halo and hence the physics of the dark matter. Asymmetric distributions of dwarfs near infalling galaxies have been observed in the Coma cluster Conselice \& Gallagher 1998, 1999). However, the observed amplitudes of the density variations are $\delta \Sigma / \Sigma \sim 5-6$, two orders of magnitude larger than our prediction for a galaxy moving through a rich cluster, so gravitational wakes are responsible for only a minor part of this enhancement. In fact, the observed excess of dwarfs in each system is contained near or within the tidal radius of the primary galaxy, indicating that the dwarfs may be bound to the primary or may have even been stripped from the primary due to ram pressure or strong tidal interactions with nearby galaxies Conselice \& Gallagher 1998). Achieving observable signal-to-noise ratios on larger scales will most likely require targeted observations of the wakes of groups falling into clusters.

For an impact parameter $b$ outside the cluster core, $\Sigma \propto\left(1+b^{2} / r_{c}^{2}\right)^{-1 / 2}$, while $\delta \Sigma \propto$ $R_{0} n(b) \propto\left(1+b^{2} / r_{c}^{2}\right)^{-1 / 2}$. Therefore the relative signal is roughly independent of position throughout the cluster, although the absolute mass density (and hence the induced convergence and shear in lenses) will decrease as $b$ increases. The increased number of cluster galaxies outside the core may make the envelopes more amenable to a statistical analysis. 


\subsection{Surface Brightness Maps}

Even without mass maps, it is possible to use observations of the ICM gas wake to constrain the dark matter properties. The ICM gas is best observed with X-rays, in which the emitted surface brightness is $S=\int\left(\epsilon_{f f} / 4 \pi\right) d \ell$. Here $\epsilon_{f f} \propto \rho_{g}^{2} T^{1 / 2}$ is the bremsstrahlung (free-free) volume emissivity. We assume in the following that the temperature of the gas varies adiabatically with its density $\left(T \propto \rho^{2 / 3}\right)$, because recent studies have shown that heat conduction is suppressed in the ICM (Vikhlinin, Markevitch, Forman, \& Jones 2001). Holding the gas isothermal decreases the signal by a small amount (see below) but does not significantly affect the structure of the observed feature.

In searching for the wake, one may look for an abrupt drop in surface brightness at the edge of its cone. An estimation of the signal strength in this case is less straightforward analytically, because the surface brightness of the Mach cone produced by a point mass diverges (due to the singularity at the edge of the cone). Fortunately, the singularity is smoothed out by an extended perturber, and far from the perturber $\Delta_{\text {gas }} \ll 1$ throughout the cone. In this region, we can expand the $\left(1+\Delta_{\text {gas }}\right)^{n}$ that appears in the emissivity and retain only the first-order term. Here $n=7 / 3$ if the gas temperature varies adiabatically with density and $n=2$ if the gas is isothermal. We then find that

$$
\left.\left.\frac{\delta S}{S}\right|_{\mathrm{FDM}} \sim\left[\frac{(\pi / 2+1) n(2 n-1)}{2 n-1+2 F(n-1 / 2, n, n+1 / 2,-1)}\right] \frac{\delta \Sigma}{\Sigma}\right|_{\mathrm{FDM}},
$$

with the same assumptions as in equation (28). Here $F(x, y, z, p)$ is the Gauss hypergeometric function (see Gradshteyn \& Ryzhik 2000, §9.10). The numerical factor is $\approx 4$ (or 5 ) if $n=2$ (or $7 / 3$ ). As with the mass excess, the amplitude of the CDM wake is enhanced over the FDM wake by a factor $\sim \alpha_{\text {FDM }}^{-1}$.

Equation (30) shows that the best targets for observations are the same as for lensing, either galaxies in groups or mergers of rich groups with clusters. If the dark matter crosssection increases with decreasing particle velocity as recently suggested Hogan \& Dalcanton 2000; Wyithe, Turner, \& Spergel 2000), galaxy groups may allow us to probe a more stronglyinteracting regime that is closer to the FDM model.

The bottom two panels in Figure 1 show the predicted surface brightness of the merging group and cluster described in the previous subsection. The wake from a halo moving through FDM (or CDM) is shown on the left (or right). The horizontal dashed lines again mark the points at which the central Mach cone intersects the cluster core. Note that the density enhancement is in fact greater than unity for $0<z<1.5$ and within the central Mach cone in the CDM case; however, the linear approximation remains valid in the remainder of this panel and in the left panel. 
Figure 2 shows analogous surface brightness maps for galaxies moving through a group with $\sigma_{c}=500 \mathrm{~km} \mathrm{~s}^{-1}, n_{c}=2 \times 10^{-3} \mathrm{~cm}^{-3}$, and $r_{c}=0.3 \mathrm{Mpc}$ : the left panels show the wake for a galaxy similar to the Milky Way, with $\sigma_{g}=170 \mathrm{~km} \mathrm{~s}^{-1}$, and the right panels show the wake from a typical massive elliptical, with $\sigma_{g}=200 \mathrm{~km} \mathrm{~s}^{-1}$. Both galaxies have $\mathcal{M}=1.5$. Results for the FDM model are shown in the top panels while those for the CDM model are shown in the bottom panels. Horizontal dashed lines show where the central Mach cone intersects the edge of the group core. Note that the maps do not include emission from the hydrodynamic wake, stripped gas, or gas still bound to the perturber. We have also ignored any possible separation between the dark and gaseous halos (see §3.2).

The sharp gradients near the central Mach cone in the CDM panels are not due to shocks (which we do not include in our formalism) but are instead due to the assumption of a singular core for the perturber. In a realistic case, the perturber will have a finite core, which smooths the gradient over the diameter of the core. To illustrate this effect, we show in Figure 3 the wake due to a uniform density perturber in the FDM (left panel) and CDM (right panel) models. The parameters are chosen identically to the right panels of Figure 2 , with the masses normalized to that of the corresponding isothermal sphere case. The smoothing of the central gradient by an extended core should help distinguish CDM wakes from the sharp cones expected in the FDM model. The morphological differences between the singular and smoothed cores are much less dramatic in the FDM case because the halos are truncated at much smaller radii in that case.

Limits on the nature of the dark matter from surface brightness maps are less direct than those from lensing maps, because we must use the ICM gas wake to infer the size of the dark matter halo. The first observational constraint comes from the width of the edge of the gravitational Mach cone, which is in turn determined by $R_{g}$. Let the observable size of the gaseous halo be $R_{\text {gas }}$. For FDM, we expect that $R_{g} \approx R_{\text {gas }}$ so that the edge of the cone is tight and follows closely the observable edge of the perturber. If, on the other hand, the dark matter is collisionless, $R_{g} \approx \alpha_{\text {gas }}^{-1} R_{\text {gas }} \gg R_{\text {gas }}$, and the edge of the gravitational cone is very extended. However, because the ICM gas is collisional, we expect hydrodynamic and stripping wakes to be present as well. The characteristic widths of these wakes is $\sim R_{\text {gas }}$, so we expect a well-defined cone to trail behind the gaseous halo even in the CDM model.

Fortunately, there remain signatures in the surface brightness distribution that can differentiate between the FDM and CDM models. First, stripped gas can be observationally distinguished from ICM gas through measurements of metallicity or gas temperature (the stripped gas will presumably be cooler and more metal-rich than the ICM gas). The hydrodynamic wake, like the gravitational wake, is composed of perturbed ICM gas, which will, if anything, be heated by compression in the wake. Therefore we can separate these two wakes 


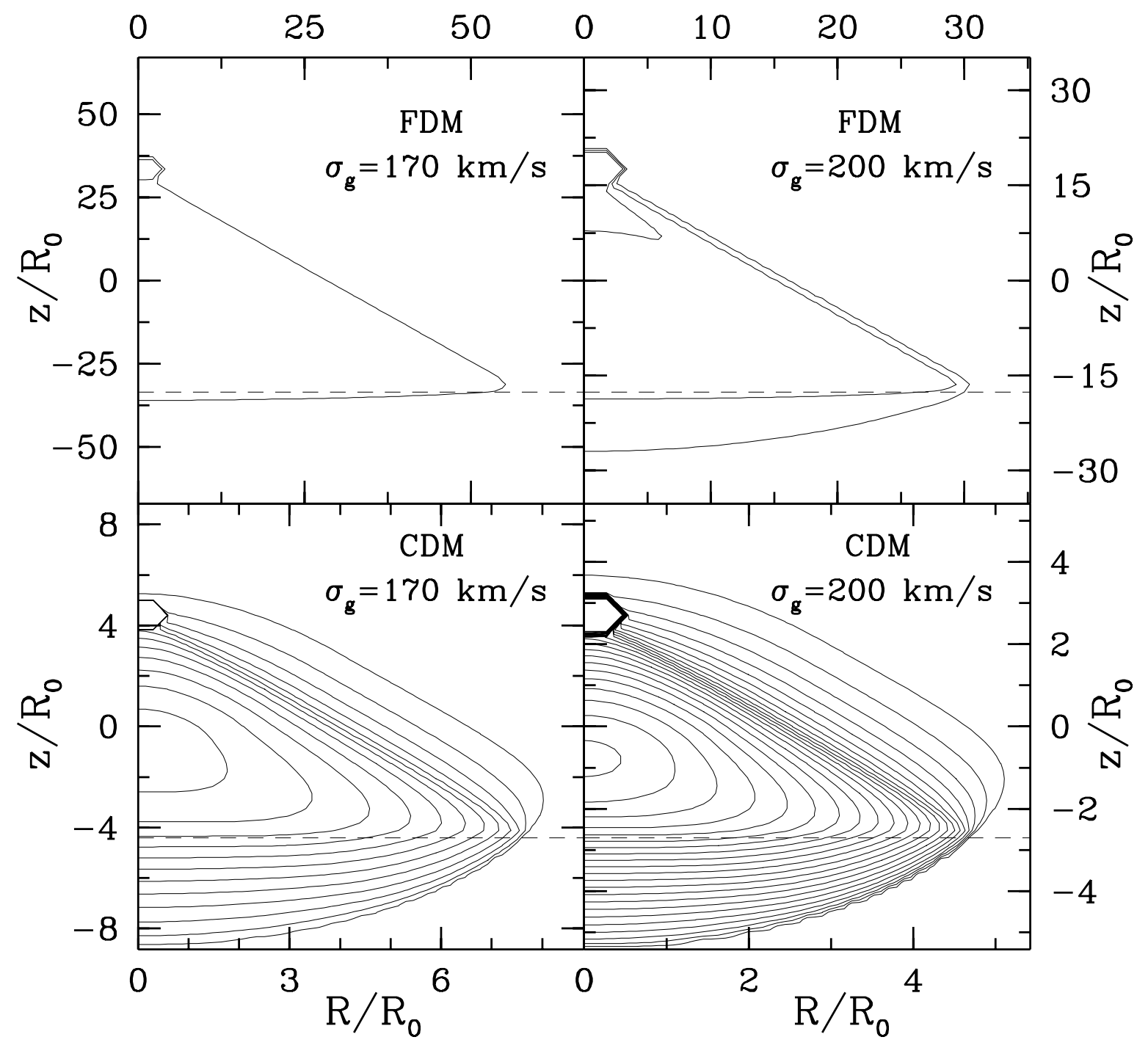

Fig. 2.- X-ray surface brightness maps for galaxies moving with $\mathcal{M}=1.5$ through a background cluster with $\sigma_{c}=500 \mathrm{~km} \mathrm{~s}^{-1}, n_{c}=2 \times 10^{-3} \mathrm{~cm}^{-3}$ and $r_{c}=0.3 \mathrm{Mpc}$. Top left: $\sigma_{g}=170 \mathrm{~km} \mathrm{~s}^{-1}$, FDM model. Top right: $\sigma_{g}=200 \mathrm{~km} \mathrm{~s}^{-1}$, FDM model. Bottom left: $\sigma_{g}=170 \mathrm{~km} \mathrm{~s}^{-1}$, CDM model. Bottom right: $\sigma_{g}=200 \mathrm{~km} \mathrm{~s}^{-1}$, CDM model. Contours are set at $5 \%$ of the peak surface brightness of the background cluster in each panel. The merger occurs in the plane of the sky with the perturber moving along the $z$-axis. Axes (in cylindrical coordinates) are in units of $R_{0}$ appropriate to each panel (see Eq. [19]). Horizontal dashed lines mark the intersection of the Mach cone with the cluster core. 


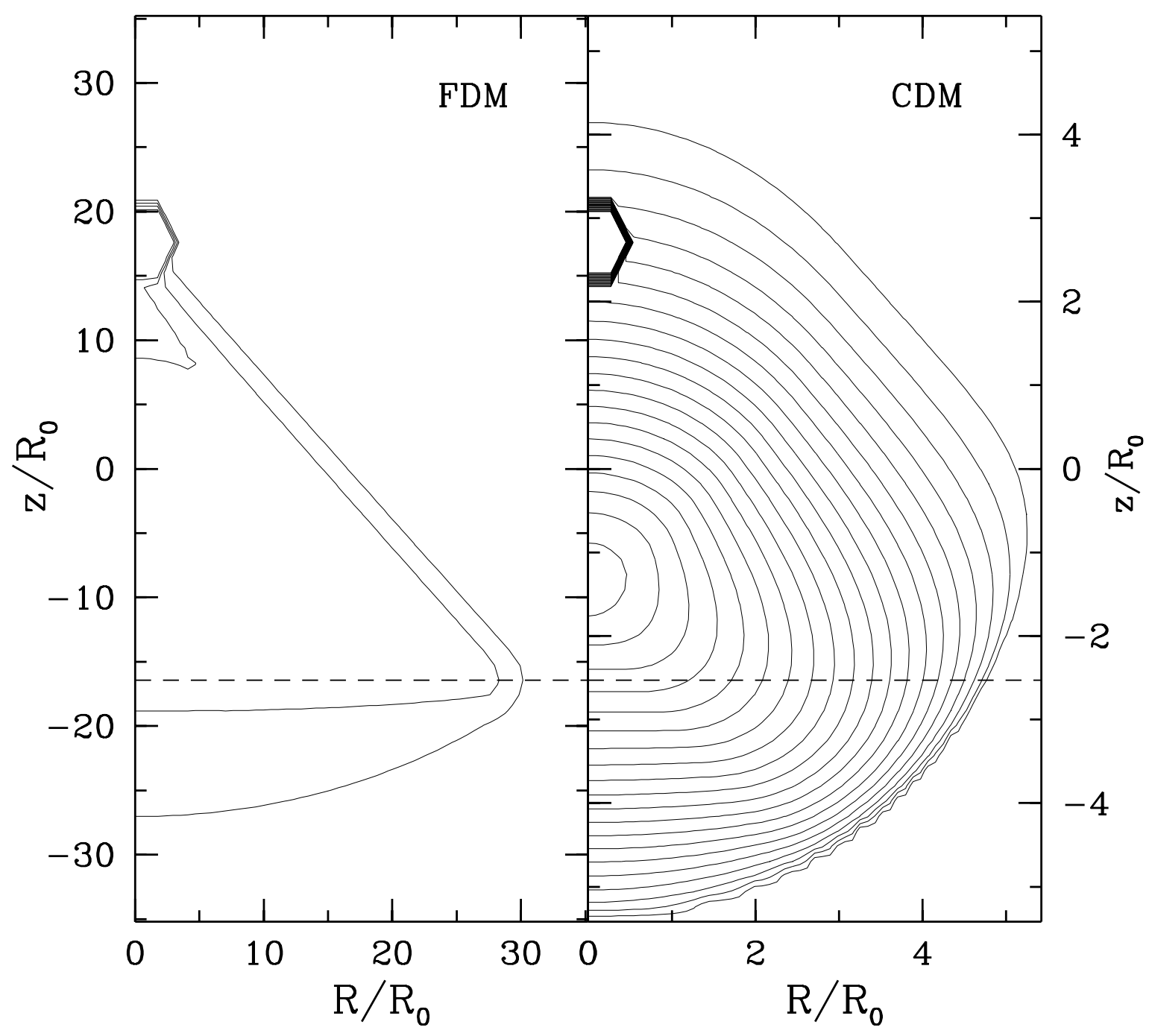

Fig. 3.- X-ray surface brightness maps for galaxies moving with $\mathcal{M}=1.5$ through a background cluster with $\sigma_{c}=500 \mathrm{~km} \mathrm{~s}^{-1}, n_{c}=2 \times 10^{-3} \mathrm{~cm}^{-3}$ and $r_{c}=0.3 \mathrm{Mpc}$. The perturber is assumed to have a uniform density, with total mass normalized to a singular isothermal sphere of $\sigma_{g}=200 \mathrm{~km} \mathrm{~s}^{-1}$. Left: FDM model. Right: CDM model. Contours are set at $5 \%$ of the peak surface brightness of the background cluster. The merger occurs in the plane of the sky with the perturber moving along the $z$-axis. Axes (in cylindrical coordinates) are in units of $R_{0}$ appropriate to each panel (see Eq. [19]). Horizontal dashed lines mark the intersection of the Mach cone with the cluster core. 
from contamination by the stripped gas.

Even without such a separation, a robust indicator of the CDM model is a surface brightness enhancement leading the hydrodynamic bow shock. Because FDM halos are the same size as the gas halo, causality dictates that their effects be confined behind the bow shock leading this body. The distance between the bow shock and the front surface of the body may be estimated if the Mach number and geometry are known (Vikhlinin, Markevitch, \& Murray 2001; Schreier 1982). Any enhancement leading this shock must be due to the gravitational effect of a CDM halo. Furthermore, in the CDM model, the gas halo may trail the dark matter halo because the latter does not experience ram pressure. In this case we would observe the gravitational brightness enhancement to be displaced even farther in front of the hydrodynamic Mach cone.

Abell 2142 is again a prime candidate for observations. Our model predicts a surface brightness excess $\delta S / S \sim 0.4-0.5$ in an FDM cone behind the object and a peak excess $\delta S / S>1$ in the CDM model, which should be easily observable in deep observations with Chandra. Galactic candidates also exist. NGC 1404, an elliptical galaxy with $\sigma_{g} \sim 150 \mathrm{~km} \mathrm{~s}^{-1}$ (Winsall \& Freeman 1993) appears to be falling into the core of the Fornax group, which has $\sigma_{c} \sim 370 \mathrm{~km} \mathrm{~s}^{-1}$ (Drinkwater, Gregg, \& Colless 2001). The infall velocity of the system is $\mathcal{M} \gtrsim 1.1$ (Ferguson 1989). Using the X-ray data of Jones et al. (1997), we predict $\delta S / S \sim 0.12-0.15\left(r_{c} / 125 \mathrm{kpc}\right)$ in the FDM model, where $r_{c}$ is the assumed core radius of the group. The peak brightness excess in the CDM model is expected to be $\delta S / S \sim 0.6\left(r_{c} / 125 \mathrm{kpc}\right)$. Paolillo et al. (2001) have made deep ROSAT observations of this system, and they see evidence for a small plume behind the galaxy. They do not see evidence for any extended cone emission, although the level we predict is equal to only one of their contours. This system therefore provides some weak evidence against the FDM model. The morphology is more consistent with a CDM wake, especially because there appears to be substantial emission leading the core of the galaxy. However, higher resolution observations are necessary in order to precisely determine the ram pressure edge of the galaxy. Furthermore, the trailing plume is not as extended as one would expect for the CDM case, although projection effects may disguise the wake.

\subsection{Comparison to Numerical Simulations}

Numerous groups have simulated cluster mergers (e.g., Roettiger, Loken, \& Burns 1997; Ricker 1998; Ricker \& Sarazin 2001; Ritchie \& Thomas 2001) and galaxy/ICM interactions (e.g., Stevens et al. 1999; Quilis et al. 2000) over the past several years. Unfortunately, neither of these classes of simulations is ideal for analysis of the gravitational wakes. In the 
latter case, the simulation box size is on the order of the galactic diameter, where nonlinear effects are strongest and where stripped material dominates the wake. In the former case, the mergers studied are generally in the nonlinear regime (most commonly, two equal mass clusters collide) and in complicated environments from which it is difficult to isolate the effects of the gravitational wake.

In fact, our formalism does not apply to existing studies of merging clusters in the CDM model because the background density varies rapidly. Such simulations (Roettiger, Loken, \& Burns 1997; Ricker \& Sarazin 2001; Ritchie \& Thomas 2001) have $\tilde{r}_{c} \sim 1$, so our formalism fails in all of these cases, even if linear theory would otherwise be valid (see equation [27]). In order to test our model and evaluate the importance of shocks and stripped gas, we suggest simulations with relatively uniform backgrounds fulfilling the criterion $\tilde{r}_{c} \gg 1$.

Recent simulations also show that shocks become significantly less important as the mass of the perturber decreases because the increase in the entropy of the core gas is small for such mergers (Ritchie \& Thomas 2001). Roettiger, Loken, \& Burns (1997) also find that gradients in the Mach number of the gas decrease as the mass ratio of the merger decreases, indicating again that shocks become significantly weaker. We therefore expect linear theory to be an adequate approximation in the limit of a small perturber mass.

To date, there has been little investigation of the direct effects of cluster mergers in the FDM regime. Ricker (1998) simulated the merger of two equally massive gaseous halos, but we do not expect linear theory to apply in this case. We therefore cannot use existing simulations to test the accuracy of our predictions for the FDM model.

A complementary set of simulations to those discussed above have been performed by Sánchez-Salcedo \& Brandenburg (1999, 2001), who investigated the accuracy of the dynamical friction force found by Ostriker (1999) for a point source traveling through a collisional medium (equation [8]). They do not directly compare the predicted and simulated wake structures, but they do find that the drag force expected from linear theory is accurate to within several percent of the simulation results, provided that the Coulomb logarithm is chosen appropriately (Sánchez-Salcedo \& Brandenburg 1999). They have also studied the drag force on a perturber in a spherically symmetric Plummer model potential (Sánchez-Salcedo \& Brandenburg 2001). In their model, $\tilde{r}_{c} \sim 6$, so we would expect our formalism to be marginally valid. Again, they do not analyze the wake structure, but they do find that the drag force on the perturber is approximately that given by linear theory if the characteristics of the background medium at the instantaneous location of the perturber are used. However, they do not include the hydrodynamic effects discussed in $\S 2.3$, so we cannot evaluate their importance relative to gravitational effects. 


\section{Discussion}

We have calculated the gravitational density wake of a perturber moving through a uniform collisional or collisionless medium. We also described how the dark matter of a galaxy or group moving through a cluster will be stripped in the cases of fluid-like dark matter (FDM) and collisionless dark matter (CDM): the former experiences ram pressure stripping, while the latter undergoes tidal stripping only. Finally, we calculated the effects of the wakes in the two different regimes on both the projected cluster mass and the X-ray surface brightness.

In the FDM model, a supersonic perturber generates a dark matter wake with a welldefined Mach cone in which the surface density increases substantially in a relatively narrow region; in the CDM model, the surface density increases more slowly over a broad region but is of larger amplitude. Because the intracluster gas is collisional, we expect the gravitational wake in the gas to form a Mach cone in both the FDM and CDM models. Nevertheless, because FDM halos are truncated at much smaller radii, the wakes in the two models have significant morphological differences that can be used to distinguish them.

We found that the signals due to these wakes are potentially observable if the perturber moves supersonically. For a large elliptical galaxy $\left(\sigma_{g} \sim 250 \mathrm{~km} \mathrm{~s}^{-1}\right)$ in a rich cluster core $\left(\sigma_{c} \sim 1000 \mathrm{~km} \mathrm{~s}^{-1}, n_{c} \sim 5 \times 10^{-3} \mathrm{~cm}^{-3}, r_{c} \sim 0.4 \mathrm{Mpc}\right)$, the variation in the surface density is $\delta \Sigma / \Sigma \sim 0.7 \%$ across the edge of the FDM Mach cone, while the variation in surface brightness is $\delta S / S \sim 3-4 \%$ for the FDM model. In the CDM model, we expect a more diffuse wake with signal strength $\sim 6$ times those in the FDM model. Fortunately, both signals increase rapidly with the relative velocity dispersions of the perturber and cluster (see equation 28]). Therefore, the most favorable systems for observations have a large $\sigma_{g} / \sigma_{c}$ : either a gas-rich group moving through a cluster or a galaxy moving in a group. For example, the expected signals for a typical galaxy $\left(\sigma_{g} \sim 170 \mathrm{~km} \mathrm{~s}^{-1}\right)$ in a galaxy group $\left(\sigma_{c} \sim 500 \mathrm{~km} \mathrm{~s}^{-1}, n_{c} \sim 2 \times 10^{-3} \mathrm{~cm}^{-3}, r_{c} \sim 0.3 \mathrm{Mpc}\right)$ are $\delta \Sigma / \Sigma \sim 1.3 \%$ and $\delta S / S \sim 7 \%$ in the FDM model (or $\delta \Sigma / \Sigma \sim 6 \%$ and $\delta S / S \sim 60 \%$ in the CDM model). Statistical methods may be used to enhance the signal-to-noise ratio; a related technique, used on ROSAT data to search for small-scale wakes due to ram pressure stripping, has been developed by Drake et al. (2000). The relative signal strength is insensitive to the location within the cluster, so it may be possible to perform such a statistical analysis over a large volume with sufficiently deep imaging.

The resolution and sensitivity of the Chandra satellite allows detailed study of the interactions between individual subhalos and the ICM. First, surface brightness and temperature observations on both sides of the bow shock leading the perturber (caused by hydrodynamic

effects unrelated to the gravitational wake; see $\S 2.3$ ) yield the Mach number of the perturber 
through the shock jump conditions (e.g., Landau \& Lifshitz 1959). We can combine this information with the observed radial velocity of the perturber or with the opening angle of the shock at large distances to find the inclination angle of the cone. When combined with optical observations (yielding $\sigma_{g}$ and $\sigma_{c}$ ) and X-ray observations (yielding $n_{c}, r_{c}$, and gas temperature), we can make an unambiguous prediction of the enhancement expected from the gravitational wake for the FDM and CDM models. This does not include the enhancement due to stripped gas or deflection of the ICM particles, but for each model it gives a lower limit to the expected enhancement. Therefore, observations showing enhancements smaller than those predicted by the CDM model can be used to falsify that model. In addition, temperature maps differentiating the cool stripped gas from hot ICM gas can help to constrain the cause of any particular observed enhancement.

Existing X-ray maps of cluster mergers and galaxy motion in groups have shown no evidence for the sharply defined Mach cones that would be expected in the FDM model (e.g., Paolillo et al. 2001); however, there is very little existing data of a high enough quality to address this question. The new generation of X-ray satellites have the resolution and sensitivity to observe these features with deep observations.

Our model predicts two systematic variations in the wakes. First, the extent of the galaxy halos should increase with distance from the cluster center: both tidal truncation (for CDM halos) and ram pressure stripping (for FDM halos) become more effective as the ambient density increases. We expect $R_{g} \propto n^{-1 / 2}$ in both cases, so this systematic variation cannot be used to test the nature of dark matter, but it can be used as a consistency check on statistical results for halo sizes.

The second systematic trend can be used to probe the collisional nature of the dark matter. Tidal truncation depends only on the relative gravitational potentials of the perturber and background cluster and is thus independent of the velocity of the perturber. Ram pressure, on the other hand, increases with velocity. We therefore expect that halo size will decrease with increasing peculiar velocity relative to the cluster in the FDM case (where $R_{g} \propto \mathcal{M}^{-1}$; see equations [17] and [18]) but not in the CDM case.

Our model for FDM assumes that the dark matter is a perfect fluid, while many SIDM models treat the dark matter as having a mean-free-path of order the size of the system. In such cases, the structure of the wake (and hence its observability) will lay somewhere between the extreme cases described here. Unfortunately, these more moderate cases are not amenable to analytic treatment. Instead, one must make use of Monte Carlo techniques in numerical simulations (e.g., Burkert 2000; Kochanek \& White 2000) to analyze the wake structure. Such simulations would also help to address some of the other shortcomings of our model (e.g., the use of linear perturbation theory in the collisional case, the assumption 
of a uniform density background, the structure of the hydrodynamic wake, and the role of shocks; see also $\S 4.3)$.

Finally, we outlined in $\S 3$ a number of consequences of the different truncation mechanisms acting on halos in clusters, including stellar mass loss and its effects on rotation curves, the fundamental plane, and local group dwarfs, relaxation times of galaxies in clusters, and separation of the dark and gaseous components of galaxy groups. While indirect, these effects do not rely on the fluid approximation and may therefore be useful in constraining the dark matter properties between the fluid and collisionless limits.

We thank W. Forman, C. Jones, M. Markevitch, and A. Vikhlinin for very helpful comments on the manuscript. We would also like to thank P. Ricker for making unpublished simulation data available to us. This work was supported in part by NASA grants NAG

5-7039, 5-7768, and NSF grants AST-9900877, AST-0071019 for AL. SRF acknowledges the support of an NSF graduate fellowship.

\section{REFERENCES}

Adami, C., Biviano, A., \& Mazure, A. 1998, A\&A, 331, 439

Arnaboldi, M. et al. 1996, ApJ, 472, 145

Arnaboldi, M. et al. 1998, ApJ, 507, 759

Babul, A. \& Taylor, J. E. 2000, ApJ, submitted (astro-ph/0012305)

Binney, J. \& Merrifield, M. 1998, Galactic Astronomy (Princeton University Press: Princeton, NJ)

Binney, J. \& Tremaine, S. 1987, Galactic Dynamics (Princeton Unifersity Press: Princeton, NJ)

Borriello, A., \& Salucci, P. 2001, MNRAS, submitted; astro-ph/0106251

Bravo-Alfaro, H., Cayatte, V., van Gorkom, J. H., \& Balkowski, C. 2000, AJ, 119, 580

Burkert, A. 1997, ApJ, 474, L99

Burkert, A. 2000, ApJ, 534, L143

Carlson, E. D., Machacek, M. E., \& Hall, L. J. 1992, ApJ, 398, 43 
Cayatte, V., Kotanyi, C., Balkowski, C., \& van Gorkom, J. H. 1994, AJ, 107, 1003

Ciardullo, R., Jacoby, G. H., \& Dejonghe, H. B. 1993, ApJ, 414, 454

Colless, M. \& Dunn, A. M. 1996, ApJ, 458, 435

Colpi, M., \& Pallavicini, A. 1998, ApJ, 502, 150

Conselice, C. J. \& Gallagher, J. S. 1998, MNRAS, 297, L34

Conselice, C. J. \& Gallagher, J. S. 1999, AJ, 117, 75

Dale, D. A., Giovanelli, R., Haynes, M. P., Hardy, E., \& Campusano, L. E. 2001, AJ, 121, 1886

de Laix, A. A., Scherrer, R. J., \& Schaefer, R. K. 1995, ApJ, 452, 495

Drake, N., Merrifield, M. R., Sakelliou, I., \& Pinkney, J. C. 2000, MNRAS, 314, 768

Drinkwater, M. J., Gregg, M. D., \& Colless, M. 2001, ApJ, 548, L139

Ferguson, H. C. 1989, AJ, 98, 367

Flores, R., \& Primack, J. R. 1994, ApJ, 427, L1

Frenk, C. S., Evrard, A. E., White, S. D. M., \& Summers, F. J. 1996, ApJ, 472, 460

Gnedin, O. Y., \& Ostriker, J. P. 2000, astro-ph/0010436

Gradshteyn, I. S., \& Ryzhik, I. M. 2000, Tabel of Integrals, Series, \& Products (Academic Press: Boston)

Henry, J. P. \& Briel, U. G. 1996, ApJ, 472, 137

Hogan, C. J., \& Dalcanton, J. J. 2000, Phys. Rev. D, 62, 063511

Irwin, M. \& Hatzidimitriou, D. 1995, MNRAS, 277, 1354

Jones, C. et al. 1997, ApJ, 482, 143

Kashikawa, N. et al. 1998, ApJ, 500, 750

Kochanek, C. S. \& White, M. 2000, ApJ, 543, 514

Kusenko, A. \& Steinhardt, P.J. 2001, astro-ph/0106008

Landau, L. D. \& Lifshitz, E. M. 1959, Fluid Mechanics (Pergamon Press: New York) 
Magri, C., Haynes, M. P., Forman, W., Jones, C., \& Giovanelli, R. 1988, ApJ, 333, 136

Markevitch, M., et al. 2000, ApJ, 541, 542

McGuire, P. C., \& Steinhardt, P. J. 2001, astro-ph/0105567

Merritt, D. 1988, in ASP Conf. Ser. 5, The Minnesota Lectures on Clusters of Galaxies and Large-Scale Structure, ed. J. Dickey (San Francisco, ASP), 175

Mobasher, B., Guzman, R., Aragon-Salamanca, A., \& Zepf, S. 1999, MNRAS, 304, 225

Moore, B. 1996, ApJ, 461, L13

Moore, B., et al. 1999, ApJ, 524, L19

Moore, B., Gelato, S., Jenkins, A., Pearce, F. R., \& Quilis, V. 2000, ApJ, 535, L21

Mulder, W. A. 1983, A\&A, 117, 9

Natarajan, P., \& Kneib, J. 1997, MNRAS, 287, 833

Natarajan, P., Kneib, J., Smail, I., \& Ellis, R. S. 1998, ApJ, 499, 600

Navarro, J., Frenk, C., \& White, S. D. M. 1997, ApJ, 490, 493

Neumann, D. M. et al. 2001, A\&A, 365, L74

Oegerle, W. R., Hill, J. M., \& Fitchett, M. J. 1995, AJ, 110, 32

Ostriker, E. C. 1999, ApJ, 513, 252

Paolillo, M., Fabbiano, G., Peres, G., \& Kim, D.-W. 2001, in X-Ray Astronomy 2000, to appear astro-ph/0106309)

Quilis, V. , Moore, B., \& Bower, R. 2000, Science, 288, 1617

Ricker, P. M. 1998, ApJ, 496, 670

Ricker, P. M., \& Sarazin, C. L. 2001, ApJ, in press (astro-ph/0107210)

Ritchie, B. W., \& Thomas, P. A. 2001, MNRAS, submitted (astro-ph/0107374)

Roettiger, K., Loken, C., \& Burns, J. O. 1997, ApJS, 109, 307

Rubin, V. C., Waterman, A. H., \& Kenney, J. D. P. 1999, AJ, 118, 236

Sánchez-Salcedo, F. J., \& Brandenburg, A. 1999, ApJ, 522, L35 
Sánchez-Salcedo, F. J. \& Brandenburg, A. 2001, MNRAS, 322, 67

Schreier, S. 1982, Compressible Flow (New York: John Wiley \& Sons), 182-9

Spergel, D.N., \& Steinhardt, P.J. 2000, Phys. Rev. Lett., 84, 3760

Stevens, I. R., Acreman, D. M., \& Ponman, T. J. 1999, MNRAS, 310, 663

Sun, M., Murray, S. S., Markevitch, M., \& Vikhlinin, A. 2001, ApJ, submitted (astro$\mathrm{ph} / 0103103)$

Vikhlinin, A., Markevitch, M., Forman, W., \& Jones, C. 2001, ApJ, submitted (astro$\mathrm{ph} / 0102483$ )

Vikhlinin, A., Markevitch, M., \& Murray, S. S. 2001, ApJ, 551, 160

Warmels, R. H. 1988, A\&AS, 72, 19

Weinberg, M. D. 1986, ApJ, 300, 93

Whitmore, B. C., Forbes, D. A., \& Rubin, V. C. 1988, ApJ, 333, 542

Winsall, M. L. \& Freeman, K. C. 1993, A\&A, 268, 443

Wyithe, J. S. B., Turner, E. L., \& Spergel, D. N. 2000, ApJ, in press astro-ph/0007354

Yoshida, N., Springel, V., White, S. D. M., \& Tormen, G. 2000, ApJ, 535, L103 